\title{
On Waring-Goldbach Problem for Squares, Cubes and Higher Powers
}

\author{
Min Zhang* \& Jinjiang $\mathrm{Li}^{\dagger}$ \\ School of Applied Science, Beijing Information Science and Technology University* \\ Beijing 100192, P. R. China \\ Department of Mathematics, China University of Mining and Technology ${ }^{\dagger}$ \\ Beijing 100083, P. R. China
}

\begin{abstract}
Let $\mathcal{P}_{r}$ denote an almost-prime with at most $r$ prime factors, counted according to multiplicity. In this paper, we generalize the result of Vaughan [42] for ternary 'admissible exponent'. Moreover, we use the refined 'admissible exponent' to prove that, for $3 \leqslant k \leqslant 14$ and for every sufficiently large even integer $n$, the following equation

$$
n=x^{2}+p_{1}^{2}+p_{2}^{3}+p_{3}^{3}+p_{4}^{3}+p_{5}^{k}
$$

is solvable with $x$ being an almost-prime $\mathcal{P}_{r(k)}$ and the other variables primes, where $r(k)$ is defined in Theorem 1.1. This result constitutes a deepening upon that of previous results.

Keywords: Waring-Goldbach problem; Hardy-Littlewood method; sieve method; almostprime.

MR(2020) Subject Classification: 11P05, 11P32, 11P55, 11N36.
\end{abstract}

\section{Introduction and main result}

The famous Goldbach Conjecture states that every even integer $N \geqslant 6$ can be written as the sum of two odd primes, i.e.

$$
N=p_{1}+p_{2}
$$

This conjecture still remains open. The recent developments on Goldbach Conjecture can be found in $[22,23,34,37,38]$ and their references.

In view of Hua's theorem [14] on five squares of primes and Lagrange's theorem on four squares, it seems reasonable to conjecture that every sufficiently large integer satisfying some necessary congruence conditions can be written as the sum of four squares of primes, i.e.

$$
N=p_{1}^{2}+p_{2}^{2}+p_{3}^{2}+p_{4}^{2}
$$

\footnotetext{
${ }^{\dagger}$ Corresponding author.

E-mail addresses: min.zhang.math@gmail.com (M. Zhang), jinjiang.li.math@gmail.com (J. Li).
} 
However, such a conjecture is out of reach at present. For the recent developments on conjecture (1.2), one can be found in $[12,13,20,29]$ and their references.

Motivated by Hua's nine cubes of primes theorem [14], it seems reasonable to conjecture that every sufficiently large even integer is the sum of eight cubes of primes, i.e.

$$
N=p_{1}^{3}+p_{2}^{3}+p_{3}^{3}+p_{4}^{3}+p_{5}^{3}+p_{6}^{3}+p_{7}^{3}+p_{8}^{3} .
$$

But unfortunately, such a conjecture (1.3) is still out of reach at present. For the recent developments on conjecture (1.3), one can see [18, 19] and its references.

Linnik [27, 28] proved that each sufficiently large odd integer $N$ can be written as $N=$ $p+n_{1}^{2}+n_{2}^{2}$, which was firstly formulated by Hardy and Littlewood [9], where $n_{1}$ and $n_{2}$ are integers. In view of this result, it seems reasonable to conjecture that every sufficiently large integer satisfying some necessary congruence conditions is a sum of a prime and two squares of primes, i.e.

$$
N=p_{1}+p_{2}^{2}+p_{3}^{2}
$$

But current techniques lack the power to solve it. Many authors considered this problem and gave some approaches to approximate (1.4) (See $[13,14,21,24,25,30,35,45,46,47]$ ). Meanwhile, we can regard this problem as the hybrid problem of (1.1) and (1.2).

In [32], Liu considered the hybrid problem of (1.1) and (1.3), i.e.

$$
N=p_{1}+p_{2}^{3}+p_{3}^{3}+p_{4}^{3}+p_{5}^{3} .
$$

There are some approximations to (1.5). On one hand, as an approach to prove (1.5), Liu and Lü [31] proved that every sufficiently large odd integer can be written as the sum of a prime, four cubes of primes and bounded number of powers of 2 , i.e.

$$
N=p_{1}+p_{2}^{3}+p_{3}^{3}+p_{4}^{3}+p_{5}^{3}+2^{v_{1}}+2^{v_{2}}+\cdots+2^{v_{K_{1}}}
$$

and gave an acceptable value of $K_{1}$. On the other hand, Liu [32] gave another approximation to (1.5). He proved that every sufficiently large odd integer $N$ can be written in the form $N=x+p_{1}^{3}+p_{2}^{3}+p_{3}^{3}+p_{4}^{3}$, where $p_{1}, p_{2}, p_{3}, p_{4}$ are primes and $x$ is an almost-prime $\mathcal{P}_{2}$. As usual, $\mathcal{P}_{r}$ always denotes an almost-prime with at most $r$ prime factors, counted according to multiplicity. In [31], Liu and Lü also considered the hybrid problem of (1.2) and (1.3),

$$
N=p_{1}^{2}+p_{2}^{2}+p_{3}^{3}+p_{4}^{3}+p_{5}^{3}+p_{6}^{3} .
$$

In their paper, they gave an approximation to (1.6) and proved that every sufficiently large even integer can be written as the sum of two squares of primes, four cubes of primes and 211 powers of 2 , i.e.

$$
N=p_{1}^{2}+p_{2}^{2}+p_{3}^{3}+p_{4}^{3}+p_{5}^{3}+p_{6}^{3}+2^{v_{1}}+2^{v_{2}}+\cdots+2^{v_{211}} .
$$

Later, in 2017, Liu [33] proved that every sufficiently large even integer can be written as the sum of two squares of primes, three cubes of primes, one fourth power of prime and a bounded number of powers of 2 , i.e.

$$
N=p_{1}^{2}+p_{2}^{2}+p_{3}^{3}+p_{4}^{3}+p_{5}^{3}+p_{6}^{4}+2^{v_{1}}+2^{v_{2}}+\cdots+2^{v_{K_{2}}} .
$$


Also, in 2016, Cai [6] gave another approximation to (1.6), and proved that any sufficiently large even integer $N$ can be written in the form $N=x^{2}+p_{1}^{2}+p_{2}^{3}+p_{3}^{3}+p_{4}^{3}+p_{5}^{3}$, where $p_{1}, p_{2}, p_{3}, p_{4}, p_{5}$ are primes and $x$ is an almost-prime $\mathcal{P}_{3}$.

In view of the results (1.7), (1.8) and the result of Cai, in this paper, we shall give some approximations to the generalized cases of (1.6).

Theorem 1.1 For $3 \leqslant k \leqslant 14$, let $\mathscr{R}_{k}(n)$ denote the number of solutions of the equation

$$
n=x^{2}+p_{1}^{2}+p_{2}^{3}+p_{3}^{3}+p_{4}^{3}+p_{5}^{k}
$$

with $x$ being an almost-prime $\mathcal{P}_{r(k)}$ and the $p_{j}$ 's primes. Then, for every sufficiently large even integer $n$, there holds

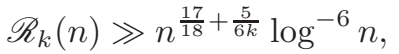

where

$$
\begin{aligned}
& r(3)=3, \quad r(4)=4, \quad r(5)=5, \quad r(6)=5, \quad r(7)=6, \quad r(8)=7 \\
& r(9)=7, r(10)=8, r(11)=9, r(12)=10, r(13)=11, r(14)=13 .
\end{aligned}
$$

We approach Theorem 1.1 via the Hardy-Littlewood method, and in a certain sense by a unified approach. To be specific, we use the ideas, which were firstly created by Brüdern $[1,2]$ and developed by Brüdern and Kawada [3, 4], combining with Hardy-Littlewood method and Iwaniec's linear sieve method to give the proof of Theorem 1.1. To treat the minor arcs in the final application of the circle method it is necessary to improve 'admissible exponents' (for the definition see Section 2) for mixed sums of cubes and $k$-th powers. In the proof of Theorem 1.1 we require a result on two cubes and a $k$-th power. The main idea is to apply the Hardy-Littlewood method as modified by Vaughan [42] to the mixed situation for one cubes and two $k$-th powers and then to combine this with the result of Vaughan [42], by the Cauchy's inequality. This auxiliary result constitutes the most novel part of the present paper which may perhaps be of interest in its own right. We formulate it precisely as Theorem 2.1 in the following section. Unfortunately Vaughan's elegant argument in [42] does not carry over very well to mixed problems; a considerable refinement of his method will be necessary. A detailed explanation is given during the proof in Section 2.

Notation. Throughout this paper, small italics denote integers when they do not obviously represent a function; $p, p_{1}, p_{2} \cdots$, with or without subscript, always stand for a prime number; $\varepsilon$ always denotes an arbitrary small positive constant, which may not be the same at different occurrences; $\gamma$ denotes Euler's constant; $f(x) \ll g(x)$ means that $f(x)=O(g(x)) ; f(x) \asymp g(x)$ means that $f(x) \ll g(x) \ll f(x)$; the constants in the $O$-term and $\ll$-symbol depend at most on $\varepsilon ; \mathcal{P}_{r}$ always denotes an almost-prime with at most $r$ prime factors, counted according to multiplicity. As usual, $\varphi(n), \mu(n)$ and $\tau_{j}(n)$ denote Euler's function, Möbius' function and the $j$-dimensional divisor function respectively. Especially, we write $\tau(n)=\tau_{2}(n)$. We denote by $a(m)$ and $b(\ell)$ arithmetical functions satisfying $|a(m)| \ll 1$ and $|b(\ell)| \ll 1 ;(s, t)$ denotes the greatest common divisor of $s$ and $t$, while $(k ; \lambda)$ is a pair of admissible exponents (see the next section); $e(\alpha)=e^{2 \pi i \alpha}$ for abbreviation. 


\section{Admissible Exponents for Cubes and Higher Powers}

The idea of admissible exponents goes back to Hardy and Littlewood [10], but was introduced formally by Davenport and Erdös [7]. Our definition is adapted from Thanigasalam [39]. let

$$
f_{k}(\alpha, X)=\sum_{X<x \leqslant 2 X} e\left(\alpha x^{k}\right)
$$

Let $k_{i} \in \mathbb{N}, 0<\lambda_{i} \leqslant 1(i=1,2, \ldots, s)$ and $P_{i}=N^{\lambda_{i} / k_{i}}$. Then the pairs

$$
\left(k_{1} ; \lambda_{1}\right),\left(k_{2} ; \lambda_{2}\right), \ldots,\left(k_{s} ; \lambda_{s}\right)
$$

are said to form admissible exponents if

$$
\int_{0}^{1}\left|f_{k_{1}}\left(\alpha, P_{1}\right) \cdots f_{k_{s}}\left(\alpha, P_{s}\right)\right|^{2} \mathrm{~d} \alpha \ll P_{1} P_{2} \cdots P_{s} N^{\varepsilon} .
$$

This is equivalent to Thanigasalam's definition, for the integral in (2.1) is equal to the number of solutions of

$$
x_{1}^{k_{1}}+x_{2}^{k_{2}}+\cdots+x_{s}^{k_{s}}=y_{1}^{k_{1}}+y_{2}^{k_{2}}+\cdots+y_{s}^{k_{s}} ; \quad P_{i}<x_{i}, y_{i} \leqslant 2 P_{i} .
$$

Our aim is to generalize the result of Vaughan [42] and establish the following Theorem.

Theorem 2.1 For $k \geqslant 4$, the pairs $(3 ; 1),\left(k ; \frac{5}{6}\right),\left(k ; \frac{5}{6}\right)$ form admissible exponents.

Proof of Theorem 2.1. Let $Q=P^{\frac{5}{2 k}}$ and let $S$ denote the number of solutions of

$$
x_{1}^{3}+y_{1}^{k}+y_{2}^{k}=x_{2}^{3}+y_{3}^{k}+y_{4}^{k}
$$

with $P<x_{i} \leqslant 2 P$ and $Q<y_{i} \leqslant 2 Q$. Then we have to show that

$$
S \ll P^{1+\varepsilon} Q^{2} .
$$

Let $S_{1}$ and $S_{2}$ denote the number of solutions of (2.2) with $x_{1}=x_{2}$ and $x_{1} \neq x_{2}$, respectively. Then, by Hua's inequality (see Lemma 2.5 of Vaughan [44]), it is easy to see that

$$
S_{1} \ll P Q^{2+\varepsilon}
$$

which is acceptable. It remains to estimate $S_{2}$. Write $x_{2}=x_{1}+h$. Then (2.2) becomes

$$
h\left(3 x_{1}^{2}+3 x_{1} h+h^{2}\right)=y_{1}^{k}+y_{2}^{k}-y_{3}^{k}-y_{4}^{k} .
$$

By symmetry it is sufficient to estimate the solutions of (2.4) with $h>0$. Since $y_{1}^{k}+y_{2}^{k} \leqslant 2^{k+1} Q^{k}$ and $x_{1}^{2}>P^{2}$, it follows that

$$
h<\frac{2^{k+1}}{3} Q^{k} P^{-2}<2^{k} Q^{k} P^{-2}=2^{k} P^{\frac{1}{2}}=H,
$$

say. Let

$$
G(\alpha)=\sum_{0<h<H} \sum_{P<x \leqslant 2 P} e\left(\alpha h\left(3 x^{2}+3 x h+h^{2}\right)\right)
$$


then

$$
S_{2} \ll \int_{0}^{1} G(\alpha)|f(\alpha)|^{4} \mathrm{~d} \alpha=\int_{\frac{1}{P H}}^{1+\frac{1}{P H}} G(\alpha)|f(\alpha)|^{4} \mathrm{~d} \alpha,
$$

where $f(\alpha)=f_{k}(\alpha, Q)$ for abbreviation. By Dirichlet's theorem on Diophantine rational approximation (for instance, see Lemma 2.1 of Vaughan [44]), each $\alpha \in[1 /(P H), 1+1 /(P H)]$ can be written in the form

$$
\alpha=\frac{a}{q}+\lambda, \quad|\lambda| \leqslant \frac{1}{q P H}
$$

for some integers $a, q$ with $1 \leqslant a \leqslant q \leqslant P H$ and $(a, q)=1$. Then we define the major arcs $\mathfrak{M}$ and minor arcs $\mathfrak{m}$ as follows:

$$
\mathfrak{M}=\bigcup_{1 \leqslant q \leqslant P} \bigcup_{\substack{1 \leqslant a \leqslant q \\(a, q)=1}} \mathfrak{M}(q, a), \quad \mathfrak{m}=\left[\frac{1}{P H}, 1+\frac{1}{P H}\right] \backslash \mathfrak{M},
$$

where

$$
\mathfrak{M}(q, a)=\left[\frac{a}{q}-\frac{1}{q P H}, \frac{a}{q}+\frac{1}{q P H}\right]
$$

Then we have

$$
\int_{\frac{1}{P H}}^{1+\frac{1}{P H}} G(\alpha)|f(\alpha)|^{4} \mathrm{~d} \alpha=\left\{\int_{\mathfrak{M}}+\int_{\mathfrak{m}}\right\} G(\alpha)|f(\alpha)|^{4} \mathrm{~d} \alpha .
$$

According to the Lemma on p. 18 of Vaughan [42], we know that

$$
G(\alpha) \ll H P^{1+\varepsilon}\left(q^{-1}+P^{-1}+q P^{-2} H^{-1}\right)^{\frac{1}{2}} .
$$

As the structure of $\mathfrak{m}$, we know that, for $\alpha \in \mathfrak{m}$, there holds $P<q \leqslant P H$, and thus

$$
G(\alpha) \ll P^{1+\varepsilon},
$$

from which and a simple consequence of Hua's lemma (Lemma 2.5 of Vaughan [44])

$$
\int_{0}^{1}|f(\alpha)|^{4} \mathrm{~d} \alpha \ll Q^{2+\varepsilon}
$$

we derive that

$$
\int_{\mathfrak{m}} G(\alpha)|f(\alpha)|^{4} \mathrm{~d} \alpha \ll P^{1+\varepsilon} Q^{2} .
$$

From (2.5), (2.7) and (2.9), we deduce that

$$
S_{2} \ll \int_{\mathfrak{M}} G(\alpha)|f(\alpha)|^{4} \mathrm{~d} \alpha+P^{1+\varepsilon} Q^{2}
$$

In order to estimate the integral on the major arcs, we approximate $G(\alpha)$ by a suitable function $G_{1}(\alpha)$. Define

$$
\begin{aligned}
& \sigma_{h}(q, a)=\sum_{x=1}^{q} e\left(\frac{a}{q}\left((x+h)^{3}-x^{3}\right)\right), \\
& v_{h}(\lambda)=\int_{P}^{2 P} e\left(\lambda\left((u+h)^{3}-u^{3}\right)\right) \mathrm{d} u, \\
& G_{1}(\alpha)=\sum_{0<h<H} q^{-1} \sigma_{h}(q, a) v_{h}\left(\alpha-\frac{a}{q}\right) .
\end{aligned}
$$


Then for $\alpha \in \mathfrak{M}(q, a), G_{1}(\alpha)$ is well defined on $\mathfrak{M}$. By (2.13) of Lemma 2 in Vaughan [43] with $k=3$, one has

$$
\sum_{P<x \leqslant 2 P} e\left(\alpha h\left(3 x^{2}+3 x h+h^{2}\right)\right)=q^{-1} \sigma_{h}(q, a) v_{h}(\lambda)+O\left(q^{\frac{1}{2}+\varepsilon}(q, h)^{\frac{1}{2}}\right),
$$

from which we obtain

$$
G(\alpha)=G_{1}(\alpha)+O\left(q^{\frac{1}{2}+\varepsilon} \sum_{0<h<H}(q, h)^{\frac{1}{2}}\right) .
$$

For the $O$-term in $(2.11)$, writing $(q, h)=d$, we see that

$$
\begin{aligned}
q^{\frac{1}{2}+\varepsilon} \sum_{0<h<H}(q, h)^{\frac{1}{2}} & \ll q^{\frac{1}{2}+\varepsilon} \sum_{d \mid q} \sum_{h<H / d} d^{\frac{1}{2}} \ll q^{\frac{1}{2}+\varepsilon} \sum_{d \mid q} d^{\frac{1}{2}} \cdot \frac{H}{d} \\
& \ll H q^{\frac{1}{2}+\varepsilon} \tau(q) \ll H q^{\frac{1}{2}+\varepsilon},
\end{aligned}
$$

from which and (2.11) we derive that

$$
G(\alpha)=G_{1}(\alpha)+O\left(P^{1+\varepsilon}\right)
$$

uniformly for $\alpha \in \mathfrak{M}$. Combining (2.8), (2.10) and (2.12), we have

$$
S_{2} \ll \int_{\mathfrak{M}} G_{1}(\alpha)|f(\alpha)|^{4} \mathrm{~d} \alpha+P^{1+\varepsilon} Q^{2} .
$$

In order to give a proper upper bound for the integral on the right-hand side of (2.13), we need to establish the following lemma, which is the crucial ingredient of this section.

Lemma 2.2 Let $\mathfrak{M}$ be defined as in (2.6), then for $k \geqslant 4$ and $X \leqslant P$, there holds

$$
\int_{\mathfrak{M}}\left|G_{1}^{2}(\alpha) f_{k}^{4}(\alpha, X)\right| \mathrm{d} \alpha \ll H P^{\varepsilon}\left(P X^{2}+X^{4}\right) .
$$

First of all, we use Lemma 2.2 to give the expected estimate of the integral on the right-hand side of (2.13) and prove it afterwards. Taking $X=Q$ and $f(\alpha)=f_{k}(\alpha, Q)$ in Lemma 2.2, then it follows from (2.8), Lemma 2.2 and Cauchy's inequality that

$$
\begin{aligned}
\int_{\mathfrak{M}} G_{1}(\alpha)|f(\alpha)|^{4} \mathrm{~d} \alpha & \ll\left(\int_{\mathfrak{M}}\left|G_{1}^{2}(\alpha) f^{4}(\alpha)\right| \mathrm{d} \alpha\right)^{\frac{1}{2}}\left(\int_{0}^{1}|f(\alpha)|^{4} \mathrm{~d} \alpha\right)^{\frac{1}{2}} \\
& \ll\left(H P^{\varepsilon}\left(P Q^{2}+Q^{4}\right)\right)^{\frac{1}{2}}\left(Q^{2+\varepsilon}\right)^{\frac{1}{2}} \ll H^{\frac{1}{2}} P^{\varepsilon}\left(P^{\frac{1}{2}} Q+Q^{2}\right) Q \\
& \ll P^{\varepsilon}\left(H^{\frac{1}{2}} P^{\frac{1}{2}} Q^{2}+H^{\frac{1}{2}} Q^{3}\right) \ll P^{1+\varepsilon} Q^{2} .
\end{aligned}
$$

From (2.3), (2.13) and (2.14), we derive the conclusion of Theorem 2.1.

Proof of Lemma 2.2. By Theorem 7.1 of Vaughan [44], it is easy to see that

$$
\sigma_{h}(q, a) \ll q^{\frac{1}{2}+\varepsilon}(q, h)^{\frac{1}{2}} .
$$

For $\alpha \in \mathfrak{M}(q, a)$, it follows from Cauchy's inequality that

$$
\left|G_{1}(\alpha)\right|^{2}=\left|\sum_{0<h<H} q^{-1} \sigma_{h}(q, a) v_{h}(\lambda)\right|^{2} \ll P^{\varepsilon}\left|\sum_{0<h<H} \frac{(q, h)^{1 / 2}}{q^{1 / 2}}\right| v_{h}(\lambda)||^{2}
$$




$$
\begin{aligned}
& \ll P^{\varepsilon}\left(\sum_{0<h<H} \frac{1}{q}\right)\left(\sum_{0<h<H}(q, h)\left|v_{h}(\lambda)\right|^{2}\right) \\
& \ll P^{\varepsilon} H q^{-1} \sum_{0<h<H}(q, h)\left|v_{h}(\lambda)\right|^{2} .
\end{aligned}
$$

By the standard estimate

$$
v_{h}(\lambda) \ll \frac{P}{1+P^{2} h|\lambda|},
$$

which combines (2.15) to give

$$
\begin{aligned}
\int_{\mathfrak{M}}\left|G_{1}^{2}(\alpha) f_{k}^{4}(\alpha, X)\right| \mathrm{d} \alpha & \ll P^{\varepsilon} H \sum_{0<h<H} \int_{\mathfrak{M}} \frac{(q, h)}{q}\left|v_{h}(\lambda)\right|^{2}\left|f_{k}^{4}(\alpha, X)\right| \mathrm{d} \alpha \\
\ll & P^{\varepsilon} H \sum_{0<h<H} \sum_{1 \leqslant q \leqslant P} \sum_{\substack{a=1 \\
(a, q)=1}}^{q} \frac{(q, h)}{q} \int_{-\frac{1}{q P H}}^{\frac{1}{q P H}}\left|v_{h}(\lambda)\right|^{2} \\
& \times \sum_{X<x_{1}, \ldots, x_{4} \leqslant 2 X} e\left(\left(\frac{a}{q}+\lambda\right)\left(x_{1}^{k}+x_{2}^{k}-x_{3}^{k}-x_{4}^{k}\right)\right) \mathrm{d} \lambda .
\end{aligned}
$$

Setting $u=x_{1}^{k}+x_{2}^{k}-x_{3}^{k}-x_{4}^{k}$, then

$$
\begin{aligned}
& \int_{\mathfrak{M}}\left|G_{1}^{2}(\alpha) f_{k}^{4}(\alpha, X)\right| \mathrm{d} \alpha \\
\ll & P^{\varepsilon} H \sum_{0<h<H} \sum_{1 \leqslant q \leqslant P} \sum_{\substack{a=1 \\
(a, q)=1}}^{q} \frac{(q, h)}{q} \sum_{u} \varrho(u) \int_{-\frac{1}{q P H}}^{\frac{1}{q P H}}\left|v_{h}(\lambda)\right|^{2} e\left(\left(\frac{a}{q}+\lambda\right) u\right) \mathrm{d} \lambda \\
\ll & P^{\varepsilon} H \sum_{0<h<H} \sum_{1 \leqslant q \leqslant P} \frac{(q, h)}{q} \sum_{u} \varrho(u)\left|\sum_{\substack{a=1 \\
(a, q)=1}}^{q} e\left(\frac{a u}{q}\right)\right| \int_{-\frac{1}{q P H}}^{\frac{1}{q P H}}\left|v_{h}(\lambda)\right|^{2} \mathrm{~d} \lambda \\
\ll & P^{\varepsilon} H \sum_{0<h<H} \sum_{1 \leqslant q \leqslant P} \frac{(q, h)}{q} \sum_{u} \varrho(u)\left|\sum_{\substack{a=1 \\
(a, q)=1}}^{q} e\left(\frac{a u}{q}\right)\right| \int_{|\lambda| \leqslant \frac{1}{P H}} \frac{P^{2}}{\left(1+P^{2} h|\lambda|\right)^{2}} \mathrm{~d} \lambda \\
\ll & P^{\varepsilon} H \sum_{0<h<H} \sum_{1 \leqslant q \leqslant P} \frac{(q, h)}{q} \sum_{u} \varrho(u)\left|\sum_{\substack{a=1 \\
(a, q)=1}}^{q} e\left(\frac{a u}{q}\right)\right| \\
\ll & \left(\int_{|\lambda| \leqslant \frac{1}{P^{2} h}} H \sum_{0<h<H} \sum_{1 \leqslant q \leqslant P} \frac{(q, h)}{q h} \sum_{u} \varrho(u) \mid \sum_{\frac{1}{P^{2} h}<|\lambda| \leqslant \frac{1}{P H}}^{\substack{q \\
P^{4} h^{2}|\lambda|^{2}}} \mathrm{~d} \lambda\right) \\
\ll & \left(\frac{a u}{q}\right) \mid,
\end{aligned}
$$

where $\varrho(u)$ denotes the number of solutions of $u=x_{1}^{k}+x_{2}^{k}-x_{3}^{k}-x_{4}^{k}$ with $X<x_{i} \leqslant 2 X(i=$ $1,2,3,4)$. By Hua's lemma (Lemma 2.5 of Vaughan [44]), we have $\varrho(0) \ll X^{2+\varepsilon}$. For $u \neq 0$, it follows from Theorem 271 of Hardy and Wright [11] that

$$
\sum_{\substack{a=1 \\(a, q)=1}}^{q} e\left(\frac{a u}{q}\right)=\sum_{d \mid(q, u)} \mu\left(\frac{q}{d}\right) d .
$$


Thus, the right-hand side of (2.16) is bounded by

$$
\begin{aligned}
& \ll P^{\varepsilon} H\left(X^{2} \sum_{0<h<H} \sum_{1 \leqslant q \leqslant P} \frac{(q, h)}{h}+\sum_{0<h<H} \sum_{1 \leqslant q \leqslant P} \frac{(q, h)}{q h} \sum_{u \neq 0} \varrho(u) \sum_{d \mid(q, u)} d\right) \\
& \ll P^{\varepsilon} H\left(\Sigma_{1}+\Sigma_{2}\right),
\end{aligned}
$$

say. Writing $r=(q, h)$, then $q=r q_{1}, h=r h_{1}$ with $\left(q_{1}, h_{1}\right)=1$. Thus, we have

$$
\begin{aligned}
\Sigma_{1} & \ll X^{2} \sum_{1 \leqslant r \leqslant P} \sum_{1 \leqslant h_{1}<H / r} \sum_{1 \leqslant q_{1} \leqslant P / r} \frac{1}{h_{1}} \\
& \ll X^{2}\left(\sum_{1 \leqslant r \leqslant P} \frac{P}{r}\right)\left(\sum_{1 \leqslant h_{1} \leqslant H / r} \frac{1}{h_{1}}\right) \\
& \ll X^{2} P\left(\sum_{1 \leqslant r \leqslant P} \frac{\log (H / r)}{r}\right) \ll X^{2} P^{1+\varepsilon} .
\end{aligned}
$$

For $\Sigma_{2}$, by the same transformation, we obtain

$$
\Sigma_{2} \ll \sum_{1 \leqslant r \leqslant P} \sum_{1 \leqslant h_{1}<H / r} \sum_{1 \leqslant q_{1} \leqslant P / r} \frac{1}{q_{1} h_{1} r} \sum_{u \neq 0} \varrho(u) \sum_{d \mid\left(r q_{1}, u\right)} d .
$$

We first consider the inner double sums over $u$ and $d$, and see that $d \mid\left(r q_{1}, u\right)$ implies $d \mid u$ and $r q_{1}=d s$ for some integer $s$. Moreover, for fixed $d$ and $s$, there exist $O\left(P^{\varepsilon}\right)$ solutions of $r q_{1}=d s$ in integer variables $r$ and $q_{1}$. Hence, we deduce that

$$
\begin{aligned}
\Sigma_{2} & \ll P^{\varepsilon} \sum_{u \neq 0} \varrho(u) \sum_{d \mid u} d \sum_{s \leqslant P} \sum_{h_{1}<H} \frac{1}{h_{1} d s} \\
& \ll P^{\varepsilon} \sum_{u \neq 0} \varrho(u)\left(\sum_{d \mid u} 1\right)\left(\sum_{s \leqslant P} \frac{1}{s}\right)\left(\sum_{h_{1}<H} \frac{1}{h_{1}}\right) \\
& \ll P^{\varepsilon} \sum_{u \neq 0} \varrho(u) \ll X^{4} P^{\varepsilon} .
\end{aligned}
$$

Combining (2.16), (2.17), (2.18) and (2.19), we get the conclusion of Lemma 2.2.

From Theorem 2.1 and the Theorem of Vaughan [42], we obtain the following corollary.

Corollary 2.3 For $k \geqslant 3$, the pairs $(3 ; 1),\left(3 ; \frac{5}{6}\right),\left(k ; \frac{5}{6}\right)$ form admissible exponents.

Proof of corollary 2.3. For $k=3$, the conclusion follows from the Theorem of Vaughan [42]. For $k \geqslant 4$, by the Theorem of Vaughan [42], Theorem 2.1 and Cauchy's inequality, we deduce that

$$
\begin{aligned}
& \int_{0}^{1}\left|f_{3}\left(\alpha, N^{\frac{1}{3}}\right) f_{3}\left(\alpha, N^{\frac{5}{18}}\right) f_{k}\left(\alpha, N^{\frac{5}{6 k}}\right)\right|^{2} \mathrm{~d} \alpha \\
\ll & \left(\int_{0}^{1}\left|f_{3}\left(\alpha, N^{\frac{1}{3}}\right) f_{3}^{2}\left(\alpha, N^{\frac{5}{18}}\right)\right|^{2} \mathrm{~d} \alpha\right)^{\frac{1}{2}}\left(\int_{0}^{1}\left|f_{3}\left(\alpha, N^{\frac{1}{3}}\right) f_{k}^{2}\left(\alpha, N^{\frac{5}{6 k}}\right)\right|^{2} \mathrm{~d} \alpha\right)^{\frac{1}{2}} \\
\ll & \left(N^{\frac{1}{3}+\frac{5}{18}+\frac{5}{18}+\varepsilon}\right)^{\frac{1}{2}}\left(N^{\frac{1}{3}+\frac{5}{6 k}+\frac{5}{6 k}+\varepsilon}\right)^{\frac{1}{2}} \ll N^{\frac{1}{3}+\frac{5}{18}+\frac{5}{6 k}+\varepsilon},
\end{aligned}
$$

which implies $(3 ; 1),\left(3 ; \frac{5}{6}\right),\left(k ; \frac{5}{6}\right)$ form admissible exponents for $k \geqslant 4$. 


\section{Proof of Theorem 1.1: Preliminaries}

In this section, we shall give some notations and preliminary lemmas. We always denote by $\chi$ a Dirichlet character $(\bmod q)$, and by $\chi^{0}$ the principal Dirichlet character $(\bmod q)$. Let

$$
\begin{aligned}
& A=10^{200}, \quad Q_{0}=\log ^{50 A} n, \quad Q_{1}=n^{\frac{5}{9}-\frac{5}{6 k}+50 \varepsilon}, \quad Q_{2}=n^{\frac{4}{9}+\frac{5}{6 k}-50 \varepsilon}, \\
& D=n^{\frac{5}{8 k}-\frac{1}{24}-51 \varepsilon}, \quad z=D^{\frac{1}{3}}, \quad X_{j}=\frac{1}{2}\left(\frac{2 n}{3}\right)^{\frac{1}{j}}, \quad X_{j}^{*}=\frac{1}{2}\left(\frac{2 n}{3}\right)^{\frac{5}{6 j}}, \quad \mathscr{P}=\prod_{2<p<z} p, \\
& F_{j}(\alpha)=\sum_{X_{j}<m \leqslant 2 X_{j}} e\left(m^{j} \alpha\right), \quad f_{j}(\alpha)=\sum_{X_{j}<p \leqslant 2 X_{j}}(\log p) e\left(p^{j} \alpha\right), \quad w_{j}(\lambda)=\int_{X_{j}}^{2 X_{j}} e\left(\lambda u^{j}\right) \mathrm{d} u, \\
& F_{j}^{*}(\alpha)=\sum_{X_{j}^{*}<m \leqslant 2 X_{j}^{*}} e\left(m^{j} \alpha\right), f_{j}^{*}(\alpha)=\sum_{X_{j}^{*}<p \leqslant 2 X_{j}^{*}}(\log p) e\left(p^{j} \alpha\right), w_{j}^{*}(\lambda)=\int_{X_{j}^{*}}^{2 X_{j}^{*}} e\left(\lambda u^{j}\right) \mathrm{d} u, \\
& G_{j}(\chi, a)=\sum_{m=1}^{q} \chi(m) e\left(\frac{a m^{j}}{q}\right), \quad S_{j}^{*}(q, a)=G_{j}\left(\chi^{0}, a\right), \quad S_{j}(q, a)=\sum_{m=1}^{q} e\left(\frac{a m^{j}}{q}\right), \\
& h(\alpha)=\sum_{m \leqslant D^{2 / 3}} a(m) \sum_{s \leqslant D^{1 / 3}} b(s) \sum_{\frac{X_{2}}{m s}<t \leqslant \frac{2 X_{2}}{m s}} e\left((m s t)^{2} \alpha\right), \\
& B_{d}(q, n)=\sum_{\substack{a=1 \\
(a, q)=1}}^{q} S_{2}\left(q, a d^{2}\right) S_{2}^{*}(q, a) S_{3}^{* 3}(q, a) S_{k}^{*}(q, a) e\left(-\frac{a n}{q}\right), \\
& B(q, n)=B_{1}(q, n), \quad A_{d}(q, n)=\frac{B_{d}(q, n)}{q \varphi^{5}(q)}, \quad A(q, n)=A_{1}(q, n), \\
& \mathfrak{S}_{d}(n)=\sum_{q=1}^{\infty} A_{d}(q, n), \quad \mathfrak{S}(n)=\mathfrak{S}_{1}(n), \\
& \mathcal{J}(n)=\int_{-\infty}^{+\infty} w_{2}^{2}(\lambda) w_{3}^{2}(\lambda) w_{3}^{*}(\lambda) w_{k}^{*}(\lambda) e(-n \lambda) \mathrm{d} \lambda, \\
& \mathcal{B}_{r}=\left\{m: X_{2}<m \leqslant 2 X_{2}, m=p_{1} p_{2} \cdots p_{r}, z \leqslant p_{1} \leqslant p_{2} \leqslant \cdots \leqslant p_{r}\right\}, \\
& \mathcal{N}_{r}=\left\{m: m=p_{1} p_{2} \cdots p_{r-1}, z \leqslant p_{1} \leqslant p_{2} \leqslant \cdots \leqslant p_{r-1}, p_{1} p_{2} \cdots p_{r-2} p_{r-1}^{2} \leqslant 2 X_{2}\right\} \text {, } \\
& g_{r}(\alpha)=\sum_{\substack{X_{2}<\ell p \leqslant 2 X_{2} \\
\ell \in \mathcal{N}_{r}}} \frac{\log p}{\log \frac{X_{2}}{\ell}} e\left(\alpha(\ell p)^{2}\right), \quad \log \boldsymbol{\Xi}=\left(\log 2 X_{2}\right)\left(\log 2 X_{3}\right)^{2}\left(\log 2 X_{3}^{*}\right)\left(\log 2 X_{k}^{*}\right) \text {, } \\
& \log \Theta=\left(\log X_{3}\right)^{2}\left(\log X_{3}^{*}\right)\left(\log X_{k}^{*}\right)
\end{aligned}
$$

Lemma 3.1 For $(a, q)=1$, we have

$$
\begin{aligned}
& \text { (i) } \quad S_{j}(q, a) \ll q^{1-\frac{1}{j}} ; \\
& \text { (ii) } G_{j}(\chi, a) \ll q^{\frac{1}{2}+\varepsilon} .
\end{aligned}
$$

In particular, for $(a, p)=1$, we have

$$
\text { (iii) }\left|S_{j}(p, a)\right| \leqslant((j, p-1)-1) \sqrt{p}
$$


(iv) $\left|S_{j}^{*}(p, a)\right| \leqslant((j, p-1)-1) \sqrt{p}+1$;

(v) $S_{j}^{*}\left(p^{\ell}, a\right)=0$ for $\ell \geqslant \gamma(p)$, where

$$
\gamma(p)= \begin{cases}\theta+2, & \text { if } p^{\theta} \| j, p \neq 2 \text { or } p=2, \theta=0, \\ \theta+3, & \text { if } p^{\theta} \| j, p=2, \theta>0 .\end{cases}
$$

Proof. For (i) and (iii)-(iv), see Theorem 4.2 and Lemma 4.3 of Vaughan [44], respectively. For (ii), see Lemma 8.5 of Hua [15] or the Problem 14 of Chapter VI of Vinogradov [41]. For (v), see Lemma 8.3 of Hua [15].

Lemma 3.2 We have

$$
\begin{aligned}
& \text { (i) } \int_{0}^{1}\left|F_{2}(\alpha) F_{3}(\alpha) F_{3}^{*}(\alpha) F_{k}^{*}(\alpha)\right|^{2} \mathrm{~d} \alpha \ll n^{\frac{11}{9}+\frac{5}{3 k}}(\log n)^{c}, \\
& \text { (ii) } \int_{0}^{1}\left|f_{2}(\alpha) f_{3}(\alpha) f_{3}^{*}(\alpha) f_{k}^{*}(\alpha)\right|^{2} \mathrm{~d} \alpha \ll n^{\frac{11}{9}+\frac{5}{3 k}}(\log n)^{c+8},
\end{aligned}
$$

where $c$ is an absolute constant.

Proof. By Lemma 2.4 of Cai [5], we know that

$$
\int_{0}^{1}\left|F_{3}(\alpha) F_{3}^{*}(\alpha)\right|^{4} \mathrm{~d} \alpha \ll n^{\frac{13}{9}} .
$$

By the above estimate, Cauchy's inequality and the explicit form of Hua's inequality (see Theorem 4 on p. 19 of Hua [15]), we deduce that

$$
\begin{aligned}
& \int_{0}^{1}\left|F_{2}(\alpha) F_{3}(\alpha) F_{3}^{*}(\alpha)\right|^{2} \mathrm{~d} \alpha \\
\ll & \left(\int_{0}^{1}\left|F_{2}(\alpha)\right|^{4} \mathrm{~d} \alpha\right)^{\frac{1}{2}}\left(\int_{0}^{1}\left|F_{3}(\alpha) F_{3}^{*}(\alpha)\right|^{4} \mathrm{~d} \alpha\right)^{\frac{1}{2}} \\
\ll & \left(X_{2}^{2}\left(\log X_{2}\right)^{c}\right)^{\frac{1}{2}}\left(n^{\frac{13}{9}}\right)^{\frac{1}{2}} \ll n^{\frac{11}{9}}(\log n)^{c} .
\end{aligned}
$$

Then (i) follows from (3.1) and the trivial estimate $\left|F_{k}^{*}(\alpha)\right| \ll n^{\frac{5}{6 k}}$. Moreover, by considering the number of solutions of the underlying Diophantine equation and the result of (i), we obtain the estimate (ii). This completes the proof of Lemma 3.2.

Lemma 3.3 For $\alpha=\frac{a}{q}+\lambda$, define

$$
\begin{gathered}
\mathcal{M}(q, a)=\left(\frac{a}{q}-\frac{1}{q Q_{0}}, \frac{a}{q}+\frac{1}{q Q_{0}}\right], \\
\Delta_{k}(\alpha)=f_{k}(\alpha)-\frac{S_{k}^{*}(q, a)}{\varphi(q)} \sum_{X_{k}<m \leqslant 2 X_{k}} e\left(m^{k} \lambda\right), \\
\mathcal{W}(\alpha)=\sum_{d \leqslant D} \frac{c(d)}{d q} S_{2}\left(q, a d^{2}\right) w_{2}(\lambda),
\end{gathered}
$$

where

$$
c(d)=\sum_{\substack{d=m \ell \\ m \leqslant D^{2 / 3} \\ \ell \leqslant D^{1 / 3}}} a(m) b(\ell) \ll \tau(d) .
$$


Then we have

$$
\sum_{1 \leqslant q \leqslant Q_{0}} \sum_{\substack{a=-q \\(a, q)=1}}^{2 q} \int_{\mathcal{M}(q, a)}\left|\mathcal{W}(\alpha) \Delta_{k}(\alpha)\right|^{2} \mathrm{~d} \alpha \ll n^{\frac{2}{k}} \log ^{-100 A} n
$$

Proof. See Lemma 2.4 of Li and Cai [26].

Lemma 3.4 For $\alpha=\frac{a}{q}+\lambda$, define

$$
\mathcal{V}_{k}(\alpha)=\frac{S_{k}^{*}(q, a)}{\varphi(q)} w_{k}(\lambda)
$$

Then we have

$$
\sum_{1 \leqslant q \leqslant Q_{0}} \sum_{\substack{a=-q \\(a, q)=1}}^{2 q} \int_{\mathcal{M}(q, a)}\left|\mathcal{V}_{k}(\alpha)\right|^{2} \mathrm{~d} \alpha \ll n^{\frac{2}{k}-1} \log ^{21 A} n,
$$

and

$$
\sum_{1 \leqslant q \leqslant Q_{0}} \sum_{\substack{a=-q \\(a, q)=1}}^{2 q} \int_{\mathcal{M}(q, a)}|\mathcal{W}(\alpha)|^{2} \mathrm{~d} \alpha \ll \log ^{21 A} n,
$$

where $\mathcal{M}(q, a)$ and $\mathcal{W}(\alpha)$ are defined by (3.2) and (3.4), respectively.

Proof. See Lemma 2.5 of $\mathrm{Li}$ and Cai [26].

For $(a, q)=1,1 \leqslant a \leqslant q \leqslant Q_{2}$, define

$$
\begin{aligned}
& \mathscr{M}(q, a)=\left(\frac{a}{q}-\frac{1}{q Q_{2}}, \frac{a}{q}+\frac{1}{q Q_{2}}\right], \quad \mathscr{M}=\bigcup_{1 \leqslant q \leqslant Q_{0}^{5}} \bigcup_{\substack{1 \leqslant a \leqslant q \\
(a, q)=1}}^{\bigcup} \mathscr{M}(q, a),
\end{aligned}
$$

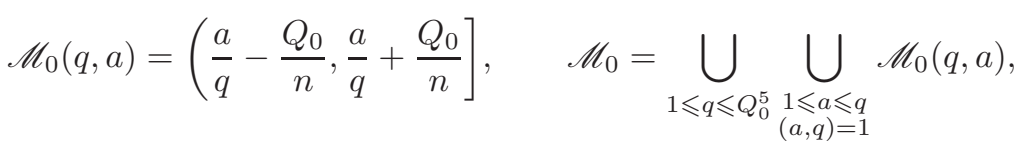

$$
\begin{aligned}
& \mathcal{I}_{0}=\left(-\frac{1}{Q_{2}}, 1-\frac{1}{Q_{2}}\right], \quad \mathfrak{m}_{0}=\mathscr{M} \backslash \mathscr{M}_{0}, \\
& \mathfrak{m}_{1}=\bigcup_{Q_{0}^{5}<q \leqslant Q_{1}} \bigcup_{\substack{1 \leqslant a \leqslant q \\
(a, q)=1}} \mathscr{M}(q, a), \quad \mathfrak{m}_{2}=\mathcal{I}_{0} \backslash\left(\mathscr{M} \cup \mathfrak{m}_{1}\right)
\end{aligned}
$$

Then we obtain the Farey dissection

$$
\mathcal{I}_{0}=\mathscr{M}_{0} \cup \mathfrak{m}_{0} \cup \mathfrak{m}_{1} \cup \mathfrak{m}_{2}
$$

Lemma 3.5 For $\alpha=\frac{a}{q}+\lambda$, define

$$
\mathcal{V}_{k}^{*}(\alpha)=\frac{S_{k}^{*}(q, a)}{\varphi(q)} w_{k}^{*}(\lambda)
$$

Then for $\alpha=\frac{a}{q}+\lambda \in \mathscr{M}_{0}$, we have

(i) $f_{j}(\alpha)=\mathcal{V}_{j}(\alpha)+O\left(X_{j} \exp \left(-\log ^{1 / 3} n\right)\right)$,

(ii) $f_{j}^{*}(\alpha)=\mathcal{V}_{j}^{*}(\alpha)+O\left(X_{j}^{*} \exp \left(-\log ^{1 / 3} n\right)\right)$, 
(iii) $g_{r}(\alpha)=\frac{c_{r}(k) \mathcal{V}_{2}(\alpha)}{\log X_{2}}+O\left(X_{2} \exp \left(-\log ^{1 / 3} n\right)\right)$,

where $\mathcal{V}_{j}(\alpha)$ is defined (3.5), and

$$
\begin{aligned}
c_{r}(k)= & (1+O(\varepsilon)) \\
& \times \int_{r-1}^{\frac{37 k-15}{15-k}} \frac{\mathrm{d} t_{1}}{t_{1}} \int_{r-2}^{t_{1}-1} \frac{\mathrm{d} t_{2}}{t_{2}} \cdots \int_{3}^{t_{r-4}-1} \frac{\mathrm{d} t_{r-3}}{t_{r-3}} \int_{2}^{t_{r-3}-1} \frac{\log \left(t_{r-2}-1\right)}{t_{r-2}} \mathrm{~d} t_{r-2} .
\end{aligned}
$$

Proof. By some routine arguments and partial summation, (i)-(iii) follow from Siegel-Walfisz theorem and prime number theorem.

Lemma 3.6 For $\alpha \in \mathfrak{m}_{2}$, we have

$$
h(\alpha) \ll n^{\frac{2}{9}+\frac{5}{12 k}-24 \varepsilon} .
$$

Proof. By the estimate (4.5) of Lemma 4.2 in Brüdern and Kawada [3], we deduce that

$$
\begin{aligned}
h(\alpha) & \ll \frac{n^{\frac{1}{2}} \tau^{2}(q) \log ^{2} n}{(q+n|q \alpha-a|)^{1 / 2}}+n^{\frac{1}{4}+\varepsilon} D^{\frac{2}{3}} \\
& \ll n^{\frac{1}{2}+\varepsilon} Q_{1}^{-\frac{1}{2}}+n^{\frac{1}{4}+\varepsilon} D^{\frac{2}{3}} \ll n^{\frac{2}{9}+\frac{5}{12 k}-24 \varepsilon},
\end{aligned}
$$

which completes the proof of Lemma 3.6.

\section{Mean Value Theorems}

In this section, we shall prove the mean value theorems for the proof of Theorem 1.1.

Proposition 4.1 For $3 \leqslant k \leqslant 14$, define

$$
J(n, d)=\sum_{\substack{m^{2}+p_{1}^{2}+p_{2}^{3}+p_{3}^{3}+p_{4}^{3}+p_{5}^{k}=n \\ X_{2}<m, p_{1} \leqslant 2 X_{2}, m=0(\bmod d) \\ X_{3}<p_{2}, p_{3} \leqslant 2 X_{3} \\ X_{3}^{*}<p_{4} \leqslant 2 X_{3}^{*} \\ X_{k}^{*}<p_{5} \leqslant 2 X_{k}^{*}}} \prod_{j=1}^{5} \log p_{j} .
$$

Then we have

$$
\sum_{m \leqslant D^{2 / 3}} a(m) \sum_{\ell \leqslant D^{1 / 3}} b(\ell)\left(J(n, m \ell)-\frac{\mathfrak{S}_{m \ell}(n)}{m \ell} \mathcal{J}(n)\right) \ll n^{\frac{17}{18}+\frac{5}{6 k}} \log ^{-A} n .
$$

Proof. Let

$$
\mathcal{K}(\alpha)=h(\alpha) f_{2}(\alpha) f_{3}^{2}(\alpha) f_{3}^{*}(\alpha) f_{k}^{*}(\alpha) e(-n \alpha) .
$$

By the Farey dissection (3.6), we have

$$
\begin{aligned}
& \sum_{m \leqslant D^{2 / 3}} a(m) \sum_{\ell \leqslant D^{1 / 3}} b(\ell) J(n, m \ell) \\
= & \int_{\mathcal{I}_{0}} \mathcal{K}(\alpha) \mathrm{d} \alpha=\left(\int_{\mathscr{M}_{0}}+\int_{\mathfrak{m}_{0}}+\int_{\mathfrak{m}_{1}}+\int_{\mathfrak{m}_{2}}\right) \mathcal{K}(\alpha) \mathrm{d} \alpha .
\end{aligned}
$$


From Hua's lemma (see Lemma 2.5 of Vaughan [44]), Corollary 2.3 and Hölder's inequality, we obtain

$$
\begin{aligned}
& \int_{0}^{1}\left|f_{2}(\alpha) f_{3}^{2}(\alpha) f_{3}^{*}(\alpha) f_{k}^{*}(\alpha)\right| \mathrm{d} \alpha \\
\ll & \left(\int_{0}^{1}\left|f_{2}(\alpha)\right|^{4} \mathrm{~d} \alpha\right)^{\frac{1}{4}}\left(\int_{0}^{1}\left|f_{3}(\alpha)\right|^{4} \mathrm{~d} \alpha\right)^{\frac{1}{4}}\left(\int_{0}^{1}\left|f_{3}(\alpha) f_{3}^{*}(\alpha) f_{k}^{*}(\alpha)\right|^{2} \mathrm{~d} \alpha\right)^{\frac{1}{2}} \\
\ll & \left(X_{2}^{2+\varepsilon}\right)^{\frac{1}{4}}\left(X_{3}^{2+\varepsilon}\right)^{\frac{1}{4}}\left(n^{\frac{1}{3}+\frac{5}{18}+\frac{5}{6 k}+\varepsilon}\right)^{\frac{1}{2}} \ll n^{\frac{13}{18}+\frac{5}{12 k}+\varepsilon} .
\end{aligned}
$$

By Lemma 3.6 and (4.2), we obtain

$$
\begin{aligned}
\int_{\mathfrak{m}_{2}} \mathcal{K}(\alpha) \mathrm{d} \alpha & \ll \sup _{\alpha \in \mathfrak{m}_{2}}|h(\alpha)| \times \int_{0}^{1}\left|f_{2}(\alpha) f_{3}^{2}(\alpha) f_{3}^{*}(\alpha) f_{k}^{*}(\alpha)\right| \mathrm{d} \alpha \\
& \ll n^{\frac{2}{9}+\frac{5}{12 k}-24 \varepsilon} \cdot n^{\frac{13}{18}+\frac{5}{12 k}+\varepsilon} \ll n^{\frac{17}{18}+\frac{5}{6 k}-23 \varepsilon} .
\end{aligned}
$$

For $\alpha \in \mathfrak{m}_{1}$, it follows from Theorem 4.1 in Vaughan [44] that

$$
h(\alpha)=\mathcal{W}(\alpha)+O\left(D Q_{1}^{\frac{1}{2}+\varepsilon}\right)=\mathcal{W}(\alpha)+O\left(n^{\frac{17}{22}+\frac{5}{24 k}-25 \varepsilon}\right),
$$

where $\mathcal{W}(\alpha)$ is defined by (3.4). Define

$$
\mathcal{K}_{1}(\alpha)=\mathcal{W}(\alpha) f_{2}(\alpha) f_{3}^{2}(\alpha) f_{3}^{*}(\alpha) f_{k}^{*}(\alpha) e(-n \alpha) .
$$

Then by (4.2) and (4.4) we have

$$
\int_{\mathfrak{m}_{1}} \mathcal{K}(\alpha) \mathrm{d} \alpha=\int_{\mathfrak{m}_{1}} \mathcal{K}_{1}(\alpha) \mathrm{d} \alpha+O\left(n^{\frac{23}{24}+\frac{5}{8 k}-24 \varepsilon}\right) .
$$

Let

$$
\begin{gathered}
\mathcal{M}_{0}(q, a)=\left(\frac{a}{q}-\frac{1}{n^{\frac{25}{36}+\frac{5}{12 k}}}, \frac{a}{q}+\frac{1}{n^{\frac{25}{36}+\frac{5}{12 k}}}\right], \quad \mathcal{M}_{0}=\bigcup_{1 \leqslant q \leqslant Q_{0}} \bigcup_{\substack{a=-q \\
(a, q)=1}}^{2 q} \mathcal{M}_{0}(q, a), \\
\mathcal{M}_{1}(q, a)=\mathcal{M}(q, a) \backslash \mathcal{M}_{0}(q, a), \quad \mathcal{M}_{1}=\bigcup_{1 \leqslant q \leqslant Q_{0}} \bigcup_{\substack{a=-q \\
(a, q)=1}}^{2 q} \mathcal{M}_{1}(q, a), \\
\mathcal{M}=\bigcup_{1 \leqslant q \leqslant Q_{0}} \bigcup_{\substack{a=-q \\
(a, q)=1}}^{2 q} \mathcal{M}(q, a),
\end{gathered}
$$

where $\mathcal{M}(q, a)$ is defined by (3.2). Then we have $\mathfrak{m}_{1} \subseteq \mathcal{I}_{0} \subseteq \mathcal{M}$. By Dirichlet's theorem on Diophantine rational approximation, we obtain

$$
\begin{aligned}
\int_{\mathfrak{m}_{1}} \mathcal{K}_{1}(\alpha) \mathrm{d} \alpha \ll & \sum_{1 \leqslant q \leqslant Q_{0}} \sum_{\substack{a=-q \\
(a, q)=1}}^{2 q} \int_{\mathfrak{m}_{1} \cap \mathcal{M}_{0}(q, a)}\left|\mathcal{K}_{1}(\alpha)\right| \mathrm{d} \alpha \\
& +\sum_{1 \leqslant q \leqslant Q_{0}} \sum_{\substack{a=-q \\
(a, q)=1}}^{2 q} \int_{\mathfrak{m}_{1} \cap \mathcal{M}_{1}(q, a)}\left|\mathcal{K}_{1}(\alpha)\right| \mathrm{d} \alpha .
\end{aligned}
$$


By Lemma 4.2 of Titchmarsh [40], we have

$$
w_{j}(\lambda) \ll \frac{X_{j}}{1+|\lambda| n},
$$

from which and the trivial estimate $\left(q, d^{2}\right) \leqslant(q, d)^{2}$, we deduce that

$$
\begin{aligned}
|\mathcal{W}(\alpha)| & \ll \sum_{d \leqslant D} \frac{\tau(d)}{d}\left(q, d^{2}\right)^{1 / 2} q^{-1 / 2}\left|w_{2}(\lambda)\right| \\
& \ll \tau_{3}(q) q^{-1 / 2}\left|w_{2}(\lambda)\right| \log ^{2} n \ll \frac{\tau_{3}(q) X_{2} \log ^{2} n}{q^{1 / 2}(1+|\lambda| n)} .
\end{aligned}
$$

Therefore, for $\alpha \in \mathcal{M}_{1}(q, a)$, we get

$$
\mathcal{W}(\alpha) \ll n^{\frac{7}{36}+\frac{5}{12 k}} \log ^{2} n,
$$

which combines (4.2) to derive that

$$
\begin{gathered}
\sum_{1 \leqslant q \leqslant Q_{0}} \sum_{\substack{a=-q \\
(a, q)=1}}^{2 q} \int_{\mathfrak{m}_{1} \cap \mathcal{M}_{1}(q, a)}\left|\mathcal{K}_{1}(\alpha)\right| \mathrm{d} \alpha \\
\ll n^{\frac{7}{36}+\frac{5}{12 k}} \log ^{2} n \times \int_{0}^{1}\left|f_{2}(\alpha) f_{3}^{2}(\alpha) f_{3}^{*}(\alpha) f_{k}^{*}(\alpha)\right| \mathrm{d} \alpha \ll n^{\frac{11}{12}+\frac{5}{6 k}+\varepsilon} .
\end{gathered}
$$

For $\alpha \in \mathcal{M}_{0}(q, a)$, it follows from Lemma 4.8 of Titchmarsh [40] that

$$
f_{3}(\alpha)=\Delta_{3}(\alpha)+\mathcal{V}_{3}(\alpha)+O(1)
$$

Hence, one obtain

$$
\begin{aligned}
& \sum_{1 \leqslant q \leqslant Q_{0}} \sum_{\substack{a=-q \\
(a, q)=1}}^{2 q} \int_{\mathfrak{m}_{1} \cap \mathcal{M}_{0}(q, a)}\left|\mathcal{K}_{1}(\alpha)\right| \mathrm{d} \alpha \\
\ll & \sum_{1 \leqslant q \leqslant Q_{0}} \sum_{\substack{a=-q \\
(a, q)=1}}^{2 q} \int_{\mathfrak{m}_{1} \cap \mathcal{M}_{0}(q, a)}\left|\mathcal{W}(\alpha) \Delta_{3}(\alpha) f_{2}(\alpha) f_{3}(\alpha) f_{3}^{*}(\alpha) f_{k}^{*}(\alpha)\right| \mathrm{d} \alpha \\
& +\sum_{1 \leqslant q \leqslant Q_{0}} \sum_{\substack{a=-q \\
(a, q)=1}}^{2 q} \int_{\mathfrak{m}_{1} \cap \mathcal{M}_{0}(q, a)}\left|\mathcal{W}(\alpha) \mathcal{V}_{3}(\alpha) f_{2}(\alpha) f_{3}(\alpha) f_{3}^{*}(\alpha) f_{k}^{*}(\alpha)\right| \mathrm{d} \alpha \\
& +\sum_{1 \leqslant q \leqslant Q_{0}} \sum_{\substack{a=-q \\
(a, q)=1}} \int_{\mathfrak{m}_{1} \cap \mathcal{M}_{0}(q, a)}\left|\mathcal{W}(\alpha) f_{2}(\alpha) f_{3}(\alpha) f_{3}^{*}(\alpha) f_{k}^{*}(\alpha)\right| \mathrm{d} \alpha \\
=: & \mathfrak{I}_{1}+\mathfrak{I}_{2}+\mathfrak{I}_{3},
\end{aligned}
$$

where $\Delta_{3}(\alpha)$ and $\mathcal{V}_{3}(\alpha)$ are defined by (3.3) and (3.5), respectively.

It follows from Cauchy's inequality, Lemma 3.2 and Lemma 3.3 that

$$
\mathfrak{I}_{1} \ll\left(\sum_{1 \leqslant q \leqslant Q_{0}} \sum_{\substack{a=-q \\(a, q)=1}}^{2 q} \int_{\mathcal{M}(q, a)}\left|\mathcal{W}(\alpha) \Delta_{3}(\alpha)\right|^{2} \mathrm{~d} \alpha\right)^{\frac{1}{2}}\left(\int_{0}^{1}\left|f_{2}(\alpha) f_{3}(\alpha) f_{3}^{*}(\alpha) f_{k}^{*}(\alpha)\right|^{2} \mathrm{~d} \alpha\right)^{\frac{1}{2}}
$$




$$
\ll\left(n^{\frac{2}{3}} \log ^{-100 A} n\right)^{\frac{1}{2}}\left(n^{\frac{11}{9}+\frac{5}{3 k}} \log ^{c+8} n\right)^{\frac{1}{2}} \ll n^{\frac{17}{18}+\frac{5}{6 k}} \log ^{-40 A} n .
$$

By (4.7), it is easy to see that, for $\alpha \in \mathfrak{m}_{1}$, there holds

$$
\sup _{\alpha \in \mathfrak{m}_{1}}|\mathcal{W}(\alpha)| \ll n^{\frac{1}{2}} \log ^{-30 A} n .
$$

Therefore, by Lemma 3.2, Lemma 3.4, (4.11) and Cauchy's inequality, we derive that

$$
\begin{aligned}
\mathfrak{I}_{2} & \ll \sup _{\alpha \in \mathfrak{m}_{1}}|\mathcal{W}(\alpha)| \cdot\left(\sum_{1 \leqslant q \leqslant Q_{0}} \sum_{\substack{a=-q \\
(a, q)=1}}^{2 q} \int_{\mathcal{M}(q, a)}\left|\mathcal{V}_{3}(\alpha)\right|^{2} \mathrm{~d} \alpha\right)^{\frac{1}{2}} \\
& \quad \times\left(\int_{0}^{1}\left|f_{2}(\alpha) f_{3}(\alpha) f_{3}^{*}(\alpha) f_{k}^{*}(\alpha)\right|^{2} \mathrm{~d} \alpha\right)^{\frac{1}{2}} \\
& \ll\left(n^{\frac{1}{2}} \log ^{-30 A} n\right) \cdot\left(n^{-\frac{1}{3}} \log ^{21 A} n\right)^{\frac{1}{2}} \cdot\left(n^{\frac{11}{9}+\frac{5}{3 k}} \log ^{c+8} n\right)^{\frac{1}{2}} \\
& \ll n^{\frac{17}{18}+\frac{5}{6 k}} \log ^{-5 A} n .
\end{aligned}
$$

It follows from Lemma 3.2 and Lemma 3.4 that

$$
\begin{aligned}
\mathfrak{I}_{3} & \ll\left(\sum_{1 \leqslant q \leqslant Q_{0}} \sum_{\substack{a=-q \\
(a, q)=1}}^{2 q} \int_{\mathcal{M}(q, a)}|\mathcal{W}(\alpha)|^{2} \mathrm{~d} \alpha\right)^{\frac{1}{2}}\left(\int_{0}^{1}\left|f_{2}(\alpha) f_{3}(\alpha) f_{3}^{*}(\alpha) f_{k}^{*}(\alpha)\right|^{2} \mathrm{~d} \alpha\right)^{\frac{1}{2}} \\
& \ll\left(\log ^{21 A} n\right)^{\frac{1}{2}} \cdot\left(n^{\frac{11}{9}+\frac{5}{3 k}} \log ^{c+8} n\right)^{\frac{1}{2}} \ll n^{\frac{11}{18}+\frac{5}{6 k}+\varepsilon} \ll n^{\frac{17}{18}+\frac{5}{6 k}-\varepsilon} .
\end{aligned}
$$

Combining (4.9), (4.10), (4.12) and (4.13), we can deduce that

$$
\sum_{1 \leqslant q \leqslant Q_{0}} \sum_{\substack{a=-q \\(a, q)=1}}^{2 q} \int_{\mathfrak{m}_{1} \cap \mathcal{M}_{0}(q, a)}\left|\mathcal{K}_{1}(\alpha)\right| \mathrm{d} \alpha \ll n^{\frac{17}{18}+\frac{5}{6 k}} \log ^{-5 A} n .
$$

From (4.5), (4.6), (4.8) and (4.14), we deduce that

$$
\int_{\mathfrak{m}_{1}} \mathcal{K}(\alpha) \mathrm{d} \alpha \ll n^{\frac{17}{18}+\frac{5}{6 k}} \log ^{-5 A} n .
$$

Similarly, we obtain

$$
\int_{\mathfrak{m}_{0}} \mathcal{K}(\alpha) \mathrm{d} \alpha \ll n^{\frac{17}{18}+\frac{5}{6 k}} \log ^{-5 A} n .
$$

For $\alpha \in \mathscr{M}_{0}$, define

$$
\mathcal{K}_{0}(\alpha)=\mathcal{W}(\alpha) \mathcal{V}_{2}(\alpha) \mathcal{V}_{3}^{2}(\alpha) \mathcal{V}_{3}^{*}(\alpha) \mathcal{V}_{k}^{*}(\alpha) e(-n \alpha) .
$$

By noticing that (4.4) still holds for $\alpha \in \mathscr{M}_{0}$, it follows from Lemma 3.5 and (4.4) that

$$
\mathcal{K}(\alpha)-\mathcal{K}_{0}(\alpha) \ll n^{\frac{35}{18}+\frac{5}{6 k}} \exp \left(-\log ^{1 / 4} n\right),
$$

which implies that

$$
\int_{\mathscr{M}_{0}} \mathcal{K}(\alpha) \mathrm{d} \alpha=\int_{\mathscr{M}_{0}} \mathcal{K}_{0}(\alpha) \mathrm{d} \alpha+O\left(n^{\frac{17}{18}+\frac{5}{6 k}} \log ^{-A} n\right) .
$$


By the well-known standard technique in the Hardy-Littlewood method, we deduce that

$$
\int_{\mathscr{M}_{0}} K_{0}(\alpha) \mathrm{d} \alpha=\sum_{m \leqslant D^{2 / 3}} a(m) \sum_{\ell \leqslant D^{1 / 3}} b(\ell) \frac{\mathfrak{S}_{m \ell}(n)}{m \ell} \mathcal{J}(n)+O\left(n^{\frac{17}{18}+\frac{5}{6 k}} \log ^{-A} n\right),
$$

and

$$
\mathcal{J}(n) \asymp n^{\frac{17}{18}+\frac{5}{6 k}} .
$$

Finally, Proposition 4.1 follows from (4.1), (4.3) and (4.15)-(4.19). This completes the proof of Proposition 4.1.

By the same method, we have the following Proposition.

Proposition 4.2 For $3 \leqslant k \leqslant 14$, define

$$
J_{r}(n, d)=\sum_{\substack{(\ell p)^{2}+m^{2}+p_{2}^{3}+p_{3}^{3}+p_{4}^{3}+p_{5}^{k}=n \\ X_{2}<\ell \leqslant 2 X_{2}, \ell \in \mathcal{N}_{r}, m \equiv 0(\bmod d) \\ X_{3}<p_{2}, p_{3} \leqslant 2 X_{3}, X_{3}^{*}<p_{4} \leqslant 2 X_{3}^{*} \\ X_{k}^{*}<p_{5} \leqslant 2 X_{k}^{*}}}\left(\frac{\log p}{\log \frac{X_{2}}{\ell}} \prod_{j=2}^{5} \log p_{j}\right) .
$$

Then we have

$$
\sum_{m \leqslant D^{2 / 3}} a(m) \sum_{t \leqslant D^{1 / 3}} b(t)\left(J_{r}(n, m t)-\frac{c_{r}(k) \mathfrak{S}_{m t}(n)}{m t \log X_{2}} \mathcal{J}(n)\right) \ll n^{\frac{17}{18}+\frac{5}{6 k}} \log ^{-A} n
$$

where $c_{r}(k)$ is defined by (3.7).

\section{On the function $\omega(d)$}

In this section, we shall investigate the function $\omega(d)$ which is defined in (5.10) and required in the proof of Theorem 1.1 .

Lemma 5.1 For $3 \leqslant k \leqslant 14$, let $\mathscr{K}(q, n)$ and $\mathscr{L}(q, n)$ denote the number of solutions of the congruences

$$
x^{2}+u_{1}^{3}+u_{2}^{3}+u_{3}^{3}+u_{4}^{k} \equiv n(\bmod q), \quad 1 \leqslant x, u_{j} \leqslant q, \quad\left(x u_{j}, q\right)=1,
$$

and

$$
x_{1}^{2}+x_{2}^{2}+u_{1}^{3}+u_{2}^{3}+u_{3}^{3}+u_{4}^{k} \equiv n(\bmod q), \quad 1 \leqslant x_{i}, u_{j} \leqslant q, \quad\left(x_{2} u_{j}, q\right)=1,
$$

respectively. Then, for all $n \equiv 0(\bmod 2)$, we have $\mathscr{L}(p, n)>\mathscr{K}(p, n)$ for all primes. Moreover, there holds

$$
\begin{aligned}
& \mathscr{L}(p, n)=p^{5}+O\left(p^{4}\right), \\
& \mathscr{K}(p, n)=p^{4}+O\left(p^{3}\right) .
\end{aligned}
$$

Proof. Let $\mathscr{L}^{*}(q, n)$ denote the number of solutions of the congruence

$$
x_{1}^{2}+x_{2}^{2}+u_{1}^{3}+u_{2}^{3}+u_{3}^{3}+u_{4}^{k} \equiv n(\bmod q), \quad 1 \leqslant x_{i}, u_{j} \leqslant q, \quad\left(x_{1} x_{2} u_{j}, q\right)=1 .
$$


Then by the orthogonality of Dirichlet characters, we have

$$
\begin{aligned}
p \cdot \mathscr{L}^{*}(p, n) & =\sum_{a=1}^{p} S_{2}^{* 2}(p, a) S_{3}^{* 3}(p, a) S_{k}^{*}(p, a) e\left(-\frac{a n}{p}\right) \\
& =(p-1)^{6}+E_{p}
\end{aligned}
$$

where

$$
E_{p}=\sum_{a=1}^{p-1} S_{2}^{* 2}(p, a) S_{3}^{* 3}(p, a) S_{k}^{*}(p, a) e\left(-\frac{a n}{p}\right) .
$$

By (iv) of Lemma 3.1, we have

$$
\left|E_{p}\right| \leqslant(p-1)(\sqrt{p}+1)^{2}(2 \sqrt{p}+1)^{3}(13 \sqrt{p}+1)
$$

It is easy to check that $\left|E_{p}\right|<(p-1)^{6}$ for $p \geqslant 19$. Hence we get $\mathscr{L}^{*}(p, n)>0$ for $p \geqslant 19$. On the other hand, for $p=2,3,5,7,11,13,17$, we can check $\mathscr{L}^{*}(p, n)>0$ directly by hand. Therefore, we obtain $\mathscr{L}^{*}(p, n)>0$ for all primes and

$$
\mathscr{L}(p, n)=\mathscr{L}^{*}(p, n)+\mathscr{K}(p, n)>\mathscr{K}(p, n) .
$$

From (5.1) and (5.2), we derive that

$$
\mathscr{L}^{*}(p, n)=p^{5}+O\left(p^{4}\right) .
$$

By a similar argument of (5.1) and (5.2), we have

$$
\mathscr{K}(p, n)=p^{4}+O\left(p^{3}\right)
$$

Combining (5.3)-(5.5), we obtain the desired results.

Lemma 5.2 The series $\mathfrak{S}(n)$ is convergent and satisfying $\mathfrak{S}(n)>0$.

Proof. From (i) and (ii) of Lemma 3.1, we obtain

$$
|A(q, n)| \ll \frac{|B(q, n)|}{q \varphi^{5}(q)} \ll \frac{q^{2+5 \varepsilon}}{\varphi^{4}(q)} \ll \frac{q^{2+5 \varepsilon}(\log \log q)^{4}}{q^{4}} \ll \frac{1}{q^{3 / 2}} .
$$

Thus, the series

$$
\mathfrak{S}(n)=\sum_{q=1}^{\infty} A(q, n)
$$

converges absolutely. Noting the fact that $A(q, n)$ is multiplicative in $q$ and by (v) of Lemma 3.1 , we get

$$
\mathfrak{S}(n)=\prod_{p}(1+A(p, n))
$$

From (iii) and (iv) of Lemma 3.1, we know that, for $p \geqslant 29$, we have

$$
|A(p, n)| \leqslant \frac{(p-1) \sqrt{p}(\sqrt{p}+1)(2 \sqrt{p}+1)^{3}(13 \sqrt{p}+1)}{p(p-1)^{5}} \leqslant \frac{200}{p^{2}} .
$$


Therefore, there holds

$$
\prod_{p \geqslant 29}(1+A(p, n)) \geqslant \prod_{p \geqslant 29}\left(1-\frac{200}{p^{2}}\right) \geqslant c_{1}>0 .
$$

On the other hand, it is easy to see that

$$
1+A(p, n)=\frac{\mathscr{L}(p, n)}{(p-1)^{5}} .
$$

By Lemma 5.1, we have $\mathscr{L}(p, n)>0$ for all $p$ with $n \equiv 0(\bmod 2)$, and thus $1+A(p, n)>0$. Consequently, we obtain

$$
\prod_{p<29}(1+A(p, n)) \geqslant c_{2}>0 .
$$

Combining (5.6), (5.7) and (5.9), we conclude that $\mathfrak{S}(n)>0$, which completes the proof of Lemma 5.2.

In view of Lemma 5.2, we define

$$
\omega(d)=\frac{\mathfrak{S}_{d}(n)}{\mathfrak{S}(n)}
$$

Similar to (5.6), we have

$$
\mathfrak{S}_{d}(n)=\prod_{p}\left(1+A_{d}(p, n)\right) .
$$

If $(d, q)=1$, then we have $S_{k}\left(q, a d^{k}\right)=S_{k}(q, a)$. Moreover, if $p \mid d$, then we get $A_{d}(p, n)=$ $A_{p}(p, n)$. Therefore, we derive that

$$
\omega(p)=\frac{1+A_{p}(p, n)}{1+A(p, n)}, \quad \omega(d)=\prod_{p \mid d} \omega(p) .
$$

Also, it is easy to show that

$$
1+A_{p}(p, n)=\frac{p}{(p-1)^{5}} \mathscr{K}(p, n) .
$$

Using (5.8), (5.12) and (5.13), we derive

$$
\omega(p)=\frac{p \cdot \mathscr{K}(p, n)}{\mathscr{L}(p, n)},
$$

from which and Lemma 5.1, we derive the following lemma.

Lemma 5.3 The function $\omega(d)$ is multiplicative and satisfies

$$
0 \leqslant \omega(p)<p, \quad \omega(p)=1+O\left(p^{-1}\right)
$$

\section{Proof of Theorem 1.1}

In this section, let $f(s)$ and $F(s)$ denote the classical functions in the linear sieve theory. Then it follows from (2.8) and (2.9) of Chapter 8 in [8] that

$$
F(s)=\frac{2 e^{\gamma}}{s}, \quad 1 \leqslant s \leqslant 3 ; \quad f(s)=\frac{2 e^{\gamma} \log (s-1)}{s}, \quad 2 \leqslant s \leqslant 4 .
$$


In the proof of Theorem 1.1, let $\lambda^{ \pm}(d)$ be the lower and upper bounds for Rosser's weights of level $D$, hence for any positive integer $d$ we have

$$
\left|\lambda^{ \pm}(d)\right| \leqslant 1, \quad \lambda^{ \pm}(d)=0 \quad \text { if } \quad d>D \quad \text { or } \quad \mu(d)=0 .
$$

For further properties of Rosser's weights we refer to Iwaniec [16]. Define

$$
\mathscr{W}(z)=\prod_{2<p<z}\left(1-\frac{\omega(p)}{p}\right) .
$$

Then from Lemma 5.3 and Mertens' prime number theorem (See [36]) we obtain

$$
\mathscr{W}(z) \asymp \frac{1}{\log N} .
$$

In order to prove Theorem 1.1, we need the following lemma.

Lemma 6.1 Under the condition (5.14), then if $z \leqslant D$, there holds

$$
\sum_{d \mid \mathscr{P}} \frac{\lambda^{-}(d) \omega(d)}{d} \geqslant \mathscr{W}(z)\left(f\left(\frac{\log D}{\log z}\right)+O\left(\log ^{-1 / 3} D\right)\right),
$$

and if $z \leqslant D^{1 / 2}$, there holds

$$
\sum_{d \mid \mathscr{P}} \frac{\lambda^{+}(d) \omega(d)}{d} \leqslant \mathscr{W}(z)\left(F\left(\frac{\log D}{\log z}\right)+O\left(\log ^{-1 / 3} D\right)\right)
$$

Proof. See Iwaniec [17], (12) and (13) of Lemma 3.

From the definition of $\mathcal{B}_{r}$, we know that $r \leqslant\left[\frac{36 k}{15-k}\right]$. Hence we obtain

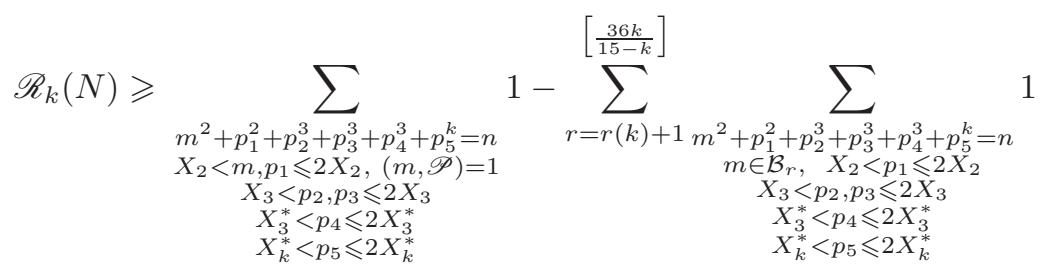

$$
\begin{aligned}
& =: \Upsilon_{0}-\sum_{r=r(k)+1}^{\left[\frac{36 k}{15-k}\right]} \Upsilon_{r} .
\end{aligned}
$$

By the property (6.2) of Rosser's weight $\lambda^{-}(d)$ and Proposition 4.1, we get

$$
\begin{aligned}
\Upsilon_{0} \geqslant \frac{1}{\log \Xi} \sum_{\substack{m^{2}+p_{1}^{2}+p_{2}^{3}+p_{3}^{3}+p_{4}^{3}+p_{5}^{k}=n \\
X_{2}<m_{,} p_{1} \leqslant 2 X_{2},(m, \mathscr{P})=1 \\
X_{3}<p_{2}, p_{3} \leqslant 2 X_{3} \\
X_{3}^{*}<p_{3} \leqslant 2 X_{3}^{*} \\
X_{k}^{*}<p_{5} \leqslant 2 X_{k}^{*}}} \prod_{j=1}^{5} \log p_{j} \\
=\frac{1}{\log \Xi} \sum_{\substack{m^{2}+p_{1}^{2}+p_{2}^{3}+p_{3}^{3}+p_{4}^{3}+p_{5}^{k}=n \\
X_{2}<m, p_{1} \leqslant 2 X_{2}, X_{3}^{*}<p_{4} \leqslant 2 X_{3}^{*} \\
X_{3}<p_{2}, p_{3} \leqslant 2 X_{3}, X_{k}^{*}<p_{5} \leqslant 2 X_{k}^{*}}}\left(\prod_{j=1}^{5} \log p_{j}\right) \sum_{d \mid(m, \mathscr{P})} \mu(d)
\end{aligned}
$$




$$
\begin{aligned}
& \geqslant \frac{1}{\log \Xi} \sum_{\substack{m^{2}+p_{1}^{2}+p_{2}^{3}+p_{3}^{3}+p_{4}^{3}+p_{5}^{k}=n \\
X_{2}<m, p_{1} \leqslant 2 X_{2}, X_{*}^{*}<p_{4} \leqslant 2 X_{3}^{*} \\
X_{3}<p_{2}, p_{3} \leqslant 2 X_{3}, X_{k}^{*}<p_{5} \leqslant 2 X_{k}^{*}}}\left(\prod_{j=1}^{5} \log p_{j}\right) \sum_{d \mid(m, \mathscr{P})} \lambda^{-}(d) \\
& =\frac{1}{\log \Xi} \sum_{d \mid \mathscr{P}} \lambda^{-}(d) J(n, d) \\
& =\frac{1}{\log \boldsymbol{\Xi}} \sum_{d \mid \mathscr{P}} \frac{\lambda^{-}(d) \mathfrak{S}_{d}(n)}{d} \mathcal{J}(n)+O\left(n^{\frac{17}{18}+\frac{5}{6 k}} \log ^{-A} n\right) \\
& =\frac{1}{\log \boldsymbol{\Xi}}\left(\sum_{d \mid \mathscr{P}} \frac{\lambda^{-}(d) \omega(d)}{d}\right) \mathfrak{S}(n) \mathcal{J}(n)+O\left(n^{\frac{17}{18}+\frac{5}{6 k}} \log ^{-A} n\right) \\
& \geqslant \frac{\mathfrak{S}(n) \mathcal{J}(n) \mathscr{W}(z)}{\log \boldsymbol{\Xi}} f(3)\left(1+O\left(\log ^{-1 / 3} D\right)\right)+O\left(n^{\frac{17}{18}+\frac{5}{6 k}} \log ^{-A} n\right) .
\end{aligned}
$$

By the property (6.3) of Rosser's weight $\lambda^{+}(d)$ and Proposition 4.2, we have

$$
\begin{aligned}
& \Upsilon_{r} \leqslant \sum_{\substack{(\ell p)^{2}+m^{2}+p_{2}^{3}+p_{3}^{3}+p_{4}^{3}+p_{5}^{k}=n \\
\ell \in \mathcal{N}_{r}, X_{2}<\ell p \leqslant 2 X_{2},(m, 9)=1 \\
X_{3}<p_{2}, p_{3} \leqslant 2 X_{3} \\
X_{3}^{*}<p_{4} \leqslant 2 X_{3}^{*} \\
X_{k}^{*}<p_{5} \leqslant 2 X_{k}^{*}}} 1 \\
& \leqslant \frac{1}{\log \Theta} \sum_{\substack{\ell p)^{2}+m^{2}+p_{2}^{3}+p_{3}^{3}+p_{4}^{3}+p_{5}^{k}=n \\
\ell \in \mathcal{N}_{r}, X_{2}<\ell p \leqslant 2 X_{2},(m, \mathscr{P})=1 \\
X_{3}<p_{2}, p_{3} \leqslant 2 X_{3} \\
X_{3}^{*}<p_{4} \leqslant 2 X_{3}^{*} \\
X_{k}^{*}<p_{5} \leqslant 2 X_{k}^{*}}} \frac{\log p}{\log \frac{X_{2}}{\ell}} \prod_{j=2}^{5} \log p_{j} \\
& =\frac{1}{\log \Theta} \sum_{\substack{(\ell p)^{2}+m^{2}+p_{2}^{3}+p_{3}^{3}+p_{4}^{3}+p_{5}^{k}=n \\
\ell \in \mathcal{N}_{r}, X_{2}<\ell p 2 X_{2} \\
X_{3}<p_{2}, p_{3} \leqslant 2 X_{3} \\
X_{3}^{*}<p_{4} \leqslant 2 X_{3}^{*} \\
X_{k}^{*}<p_{5} \leqslant 2 X_{k}^{*}}}\left(\frac{\log p}{\log \frac{X_{2}}{\ell}} \prod_{j=2}^{5} \log p_{j}\right) \sum_{d \mid(m, \mathscr{P})} \mu(d) \\
& \leqslant \frac{1}{\log \Theta} \sum_{\substack{(\ell p)^{2}+m^{2}+p_{2}^{3}+p_{3}^{3}+p_{4}^{3}+p_{5}^{k}=n \\
\ell \in \mathcal{N}_{r}, X_{2}<\ell p 2 X_{2} \\
X_{3}<p_{2}, p_{3} \leqslant 2 X_{3} \\
X_{3}^{*}<p_{4} \leqslant 2 X_{3}^{*} \\
X_{k}^{*}<p_{5} \leqslant 2 X_{k}^{*}}}\left(\frac{\log p}{\log \frac{X_{2}}{\ell}} \prod_{j=2}^{5} \log p_{j}\right) \sum_{d \mid(m, \mathscr{P})} \lambda^{+}(d) \\
& =\frac{1}{\log \Theta} \sum_{d \mid \mathscr{P}} \lambda^{+}(d) J_{r}(n, d) \\
& =\frac{1}{\log \Theta} \sum_{d \mid \mathscr{P}} \frac{\lambda^{+}(d) c_{r}(k) \mathfrak{S}_{d}(n)}{d \log X_{2}} \mathcal{J}(n)+O\left(n^{\frac{17}{18}+\frac{5}{6 k}} \log ^{-A} n\right) \\
& =\frac{c_{r}(k) \mathfrak{S}(n) \mathcal{J}(n)}{\left(\log X_{2}\right) \log \boldsymbol{\Theta}} \sum_{d \mid \mathscr{P}} \frac{\lambda^{+}(d) \omega(d)}{d}+O\left(n^{\frac{17}{18}+\frac{5}{6 k}} \log ^{-A} n\right) \\
& \leqslant \frac{c_{r}(k) \mathfrak{S}(n) \mathcal{J}(n) \mathscr{W}(z)}{\log \Xi} F(3)\left(1+O\left(\log ^{-1 / 3} D\right)\right)+O\left(n^{\frac{17}{18}+\frac{5}{6 k}} \log ^{-A} n\right) .
\end{aligned}
$$

According to simple numerical calculations, we know that

$$
c_{4}(3) \leqslant 0.4443636, c_{5}(3) \leqslant 0.0578256, c_{j}(3) \leqslant 0.0027627 \text { with } 6 \leqslant j \leqslant 9 ;
$$




$$
\begin{aligned}
& c_{5}(4) \leqslant 0.3029445, c_{6}(4) \leqslant 0.0459743, c_{j}(4) \leqslant 0.00388094 \text { with } 7 \leqslant j \leqslant 13 \\
& c_{6}(5) \leqslant 0.1892887, c_{j}(5) \leqslant 0.0307123 \text { with } 7 \leqslant j \leqslant 18 ; \\
& c_{6}(6) \leqslant 0.4867818, c_{7}(6) \leqslant 0.1133016, c_{8}(6) \leqslant 0.01913692, \\
& c_{j}(6) \leqslant 0.00237244 \text { with } 9 \leqslant j \leqslant 24 ; \\
& c_{7}(7) \leqslant 0.2978111, c_{8}(7) \leqslant 0.0672273, c_{j}(7) \leqslant 0.0117295 \text { with } 9 \leqslant j \leqslant 31 \\
& c_{8}(8) \leqslant 0.1830229, c_{9}(8) \leqslant 0.0407894, c_{j}(8) \leqslant 0.0073521 \text { with } 10 \leqslant j \leqslant 41 ; \\
& c_{8}(9) \leqslant 0.4323101, c_{9}(9) \leqslant 0.1169923, c_{10}(9) \leqslant 0.02614497, \\
& c_{11}(9) \leqslant 0.0048887, c_{j}(9) \leqslant 0.000772739 \text { with } 12 \leqslant j \leqslant 54 ; \\
& c_{9}(10) \leqslant 0.3023038, c_{10}(10) \leqslant 0.0809431, c_{11}(10) \leqslant 0.0184125, \\
& c_{j}(10) \leqslant 0.003597861 \text { with } 12 \leqslant j \leqslant 72 ; \\
& c_{10}(11) \leqslant 0.2360241, c_{11}(11) \leqslant 0.0639155, c_{12}(11) \leqslant 0.01504156, \\
& c_{j}(11) \leqslant 0.003105002 \text { with } 13 \leqslant j \leqslant 99 ; \\
& c_{11}(12) \leqslant 0.2231261, c_{12}(12) \leqslant 0.06262236, c_{13}(12) \leqslant 0.01555779, \\
& c_{14}(12) \leqslant 0.00344782, c_{j}(12) \leqslant 0.0006868855 \text { with } 15 \leqslant j \leqslant 144 \\
& c_{12}(13) \leqslant 0.2976851, c_{13}(13) \leqslant 0.0895433, c_{14}(13) \leqslant 0.0242215, \\
& c_{15}(13) \leqslant 0.005929363, c_{16}(13) \leqslant 0.0013212887, \\
& c_{j}(13) \leqslant 0.0002694412 \text { with } 17 \leqslant j \leqslant 234 ; \\
& c_{14}(14) \leqslant 0.2926583, c_{15}(14) \leqslant 0.09172191, c_{16}(14) \leqslant 0.026363835, \\
& c_{17}(14) \leqslant 0.006978431, c_{18}(14) \leqslant 0.001783123, \\
& c_{j}(14) \leqslant 0.0002510648 \text { with } 19 \leqslant j \leqslant 504 .
\end{aligned}
$$

Therefore, if we write

$$
C(k)=\sum_{r=r(k)+1}^{\left[\frac{36 k}{15-k}\right]} c_{r}(k),
$$

then we have

$$
\begin{aligned}
& C(3)<0.513241, \quad C(4)<0.376086, \quad C(5)<0.557837, \quad C(6)<0.657181 \\
& C(7)<0.634817, \quad C(8)<0.459081, \quad C(9)<0.613564, \quad C(10)<0.621131, \\
& C(11)<0.585117, C(12)<0.394051, C(13)<0.477439, \quad C(14)<0.541523 .
\end{aligned}
$$

From (6.1), (6.4)-(6.10), we derive that

$$
\begin{aligned}
\mathscr{R}_{k}(N) \geqslant & \left(f(3)-F(3) \sum_{r=r(k)+1}^{\left[\frac{36 k}{15-k}\right]} c_{r}(k)\right)\left(1+O\left(\log ^{-1 / 3} D\right)\right) \\
& \times \frac{\mathfrak{S}(n) \mathcal{J}(n) \mathscr{W}(z)}{\log \Xi}+O\left(n^{\frac{17}{18}+\frac{5}{6 k}} \log ^{-A} n\right) \\
\geqslant & \frac{2 e^{\gamma}}{3}(\log 2-0.657181)\left(1+O\left(\log ^{-1 / 3} D\right)\right)
\end{aligned}
$$




$$
\begin{aligned}
& \times \frac{\mathfrak{S}(n) \mathcal{J}(n) \mathscr{W}(z)}{\log \Xi}+O\left(n^{\frac{17}{18}+\frac{5}{6 k}} \log ^{-A} n\right) \\
& \gg n^{\frac{17}{18}+\frac{5}{6 k}} \log ^{-6} n,
\end{aligned}
$$

which completes the proof of Theorem 1.1.

\section{Acknowledgement}

The authors would like to express the most sincere gratitude to the referee for his/her patience in refereeing this paper. This work is supported by the Fundamental Research Funds for the Central Universities (Grant No. 2019QS02), and National Natural Science Foundation of China (Grant No. 11901566, 11971476).

\section{References}

[1] J. Brüdern, A sieve approach to the Waring-Goldbach problem I. Sums of four cubes, Ann. Sci. cole Norm. Sup. (4), 28 (1995), no. 4, 461-476.

[2] J. Brüdern, A sieve approach to the Waring-Goldbach problem II. On the seven cubes theorem, Acta Arith., 72 (1995), no. 3, 211-227.

[3] J. Brüdern, K. Kawada, Ternary problems in additive prime number theory, in: Analytic Number Theory, C. Jia and K. Matsumoto (eds.), Dev. Math. 6, Kluwer, Dordrecht, 2002, 39-91.

[4] J. Brüdern, K. Kawada, On the Waring-Goldbach problem for cubes, Glasg. Math. J., 51 (2009), no. 3, 703-712.

[5] Y. C. Cai, The Waring-Goldbach problem: one square and five cubes, Ramanujan J., 34 (2014), no. $1,57-72$.

[6] Y. C. Cai, Waring-Goldbach problem: two squares and higher powers, J. Théor. Nombres Bordeaux, 28 (2016), no. 3, 791-810.

[7] H. Davenport, P. Erdös, On sums of positive integral k-th powers, Ann. of Math. (2), 40 (1939), 533-536.

[8] H. Halberstam, H. E. Richert, Sieve Methods, Academic Press, London, 1974.

[9] G. H. Hardy, J. E. Littlewood, Some problems of 'Partitio numerorum' III: On the expression of a number as a sum of primes, Acta Math., 44 (1923), no. 1, 1-70.

[10] G. H. Hardy, J. E. Littlewood, Some problems of 'Partitio numerorum' (VI): Further researches in Waring's Problem, Math. Z., 23 (1925), no. 1, 1-37.

[11] G. H. Hardy, E. M. Wright, An introduction to the theory of numbers, fifth edition, Oxford University Press, New York, 1979. 
[12] G. Harman, A. V. Kumchev, On sums of squares of primes, Math. Proc. Cambridge Philos. Soc., 140 (2006), no. 1, 1-13.

[13] G. Harman, A. V. Kumchev, On sums of squares of primes II, J. Number Theory, 130 (2010), no. 9, 1969-2002.

[14] L. K. Hua, Some results in the additive prime number theory, Quart. J. Math. Oxford Ser. (2), 9 (1938), no. 1, 68-80.

[15] L. K. Hua, Additive Theory of Prime Numbers, American Mathematical Society, Providence, Rhode Island, 1965.

[16] H. Iwaniec, Rosser's sieve, Acta Arith., 36 (1980), no. 2, 171-202.

[17] H. Iwaniec, A new form of the error term in the linear sieve, Acta Arith., 37 (1980), no. $1,307-320$.

[18] K. Kawada, L. Zhao, The Waring-Goldbach problem for cubes with an almost prime, Proc. Lond. Math. Soc. (3), 119 (2019), no. 4, 867-898.

[19] A. V. Kumchev, D. I. Tolev, An invitation to additive prime number theory, Serdica Math. J., 31 (2005), no. 1-2, 1-74.

[20] A. V. Kumchev, L. Zhao, On sums of four squares of primes, Mathematika, 62 (2016), no. $2,348-361$.

[21] M. C. Leung, M. C. Liu, On generalized quadratic equations in three prime variables, Monatsh. Math., 115 (1993), no. 1-2, 133-167.

[22] H. Z. Li, The exceptional set of Goldbach numbers, Quart. J. Math. Oxford Ser. (2), 50 (1999), no. 200, 471-482.

[23] H. Z. Li, The exceptional set of Goldbach numbers II, Acta Arith., 92 (2000), no. 1, 71-88.

[24] H. Z. Li, Representation of odd integers as the sum of one prime, two squares of primes and powers of 2, Acta Arith., 128 (2007), no. 3, 223-233.

[25] H. Z. Li, Sums of one prime and two prime squares, Acta Arith., 134 (2008), no. 1, 1-9.

[26] Y. J. Li, Y. C. Cai, Waring-Goldbach problem: two squares and some higher powers, J. Number Theory, 162 (2016), 116-136.

[27] Yu. V. Linnik, Hardy-Littlewood problem on representation as the sum of a prime and two squares, Dokl. Akad. Nauk SSSR, 124 (1959), 29-30.

[28] Yu. V. Linnik, An asymptotic formula in an additive problem of Hardy-Littlewood, Izv. Akad. Nauk SSSR Ser. Mat., 24 (1960), 629-706.

[29] J. Y. Liu, T. Zhan, The quadratic Waring-Goldbach problem, J. Shandong Univ. Nat. Sci., 42 (2007), no. 2, 1-18. 
[30] T. Liu, Representation of odd integers as the sum of one prime, two squares of primes and powers of 2, Acta Arith., 115 (2004), no. 2, 97-118.

[31] Z. X. Liu, G. S. Lü, Two results on powers of 2 in Waring-Goldbach problem, J. Number Theory, 131 (2011), no. 4, 716-736.

[32] Z. X. Liu, Cubes of primes and almost prime, J. Number Theory, 132 (2012), no. 6, 1284-1294.

[33] Z. X. Liu, Goldbach-Linnik type problems with unequal powers of primes, J. Number Theory, 176 (2017), 439-448.

[34] W. C. Lu, Exceptional set of Goldbach number, J. Number Theory, 130 (2010), no. 10, $2359-2392$.

[35] G. S. Lü, H. W. Sun, Integers represented as the sum of one prime, two squares of primes and powers of 2, Proc. Amer. Math. Soc., 137 (2009), no. 4, 1185-1191.

[36] F. Mertens, Ein Beitrag zur analytyischen Zahlentheorie, J. Reine Angew. Math., 78 (1874), 46-62.

[37] J. Pintz, Landaus problems on primes, J. Théor. Nombres Bordeaux, 21 (2009), no. 2, 357-404.

[38] J. Pintz, A new explicit formula in the additive theory of primes with applications II. The exceptional set in Goldbach's problem, arXiv:1804.09084.

[39] K. Thanigasalam, On sums of powers and a related problem, Acta Arith., 36 (1980), no. $2,125-141$.

[40] E. C. Titchmarsh, The Theory of the Riemann Zeta-Function, second edition, Oxford University Press, Oxford, 1986.

[41] I. M. Vinogradov, Elements of Number Theory, Dover Publications, New York, 1954.

[42] R. C. Vaughan, Sums of three cubes, Bull. London Math. Soc., 17 (1985), no. 1, 17-20.

[43] R. C. Vaughan, On Waring's problem for smaller exponents, Proc. London Math. Soc. (3), 52 (1986), no. 3, 445-463.

[44] R. C. Vaughan, The Hardy-Littlewood Method, second edition, Cambridge University Press, Cambridge, 1997.

[45] M. Q. Wang, On the sum of a prime and two prime squares, Acta Math. Sinica (Chin. Ser.), 47 (2004), no. 5, 845-858.

[46] M. Q. Wang, X. M. Meng, The exceptional set in the two prime squares and a prime problem, Acta Math. Sin. (Engl. Ser.), 22 (2006), no. 5, 1329-1342.

[47] L. Zhao, The additive problem with one prime and two squares of primes, J. Number Theory, 135 (2014), 8-27. 